\title{
Structures of scalar transport in a turbulent channel
}

\author{
Suranga Dharmarathne ${ }^{\mathrm{a}}$, Murat Tutkun ${ }^{\mathrm{b}, \mathrm{c}}$, Guillermo Araya ${ }^{\mathrm{a}}$, Luciano \\ Castillo $^{\mathrm{a}, *}$ \\ ${ }^{a}$ Department of Mechanical Engineering, Texas Tech University, Lubbock, TX-79409, USA \\ ${ }^{b}$ Department of Process and Fluid Flow Technology, Institute for Energy Technology (IFE), \\ 2027 Kjeller, Norway \\ ${ }^{c}$ Department of Mathematics, University of Oslo, Blindern, 0316 Oslo, Norway
}

\begin{abstract}
Direct numerical simulation (DNS) of a turbulent channel with temperature treated as a passive scalar at a moderate Reynolds number $\left(R e_{\tau}=395\right)$ was performed to investigate the large scale motions responsible for scalar transport. Structures are elicited by three dimensional two-point correlations. Complete three dimensional structure of cross correlations between velocity and scalar fluctuations are evaluated for the first time. The results show that the organized motions which are responsible for transporting streamwise component of the turbulence kinetic energy, scalar variance, and streamwise heat flux are very similar only in the viscous sublayer. However, the large scale motions (LSM) which transport streamwise component of the turbulent kinetic energy, scalar variance, and streamwise heat flux are clearly distinct to each other beyond the buffer region. In addition, comprehensive three dimensional two-point correlation data imply that LSM which are responsible for carrying most of the Reynolds stresses are not exactly the same as those that transport most of the scalar fluxes in both inner and outer regions of the turbulent boundary layer. Analysis length scales of correlations revealed that the growth of LSM which influence both momentum and thermal transport is linear. Comparison of physical extents of LSM which are responsible for transporting Reynolds stresses and
\end{abstract}

\footnotetext{
${ }^{*}$ Corresponding author

Email addresses: suranga.dharmarathne@ttu.edu (Suranga Dharmarathne), murat.tutkun@ife.no (Murat Tutkun), g.araya@ttu.edu (Guillermo Araya), luciano.castillo@ttu.edu (Luciano Castillo)
}

Preprint submitted to European Journal of Mechanics B-Fluids 
wall-normal heat fluxes reveals that Reynolds analogy holds for scalar transport by the LSM in the boundary layer.

Keywords: DNS, Large-scale motions, Scalar transport, Correlation, Turbulent channel

2010 MSC: 00-01, 99-00

\section{Introduction}

Turbulent transport of passive scalars is an important realm of turbulence which has numerous applications in many natural and man-made applications (Shraiman \& Sigga, 2000). Passive scalar is a diffusive contaminant in a fluid

5 flow that exists in very low concentration so that it has no effect on the dynamics of the fluid motion (Warhaft, 2000). Even though the scalar field is passively advected by the velocity field, local isotropy and the universality of the scalar field are questionable even at the dissipative range in contrast to the velocity field (Sreenivasan, 1991) and it is well known that the LSM which are responsible for momentum transport are anisotropic. Hence, we can hypothesize that LSM which mostly contribute the momentum transport might be significantly different than that of the LSM which transport scalar.

The concept of eddies or organized motions in turbulent flows was first introduced by Townsend (1956) based on long tails of velocity correlations. This knowledge brought about a paradigm shift in turbulence research in terms of organized motions. Experimental observations of low and high speed streaks in the near-wall region by Hama (see Corrsin, 1956) and Kline et al. (1967) enhanced our fundamental knowledge of the near wall structures and events such as quasi-streamwise vortices (Bakewell \& Lumley, 1967; Blackwelder \& Eck20 elmann, 1979), hairpin vortices (Head \& Bandyopadhyay, 1981, Smith et al. 1991; Adrian, 2007), internal shear layers, sweeps, and ejections (Robinson, 1991). Dynamical significance and contribution to the self-sustaining mechanism of the wall turbulence of these structures are well documented (Panton, 2001). Furthermore, their contribution to the momentum transport has also 
been investigated extensively (Hussain, 1986).

The growing interest in the importance of the near wall structures in momentum transport evoked researchers to seek the influence of these structures on the scalar transport. For instance, Perry \& Hoffmann (1976) investigated the turbulent boundary layer with temperature as the passive scalar field. They concluded that the structures and the events which are responsible for momentum transport, promote the scalar transport as well. Iritani et al. (1985) used a water channel to observe both momentum and thermal streaks simultaneously and noticed a high correlation of momentum streaks with thermal streaks. Emergence of direct numerical simulation as a research tool enabled collecting high-fidelity data particularly in the near-wall region. Hence, near wall structures and events could be investigated in great details. Kim \& Moin (1987) confirmed the observations made by Iritani et al. (1985) using DNS of turbulent channel with different molecular Prandtl numbers $(P r)$ while Kong et al. (2000) reassured the same findings utilizing DNS for a turbulent bound-

40 ary layer with iso-flux and iso-thermal boundary boundary conditions. More recently, Abe et al. (2009) investigated the relationship between near-wall organized motions and scalar mixing. They concluded that streaks, quasi-stremwise vortices, and internal shear layers contribute to the scalar dissipation rate.

घ The first notable evidence of LSM in the outer layer was provided by Kovasz-

45 nay et al. (1970) by using two-point correlation maps in a spatially developing boundary layer at momentum thickness Reynolds number of 2750 . They suggested that LSM are related to "bulges" which plays a key role at the turbulent non-turbulent interface. Streamwise and spanwise lengths of these structures were estimated to be $2-3 \delta$ and $0.5-1 \delta$ respectively. With the recent advancements of computer resources, studies at high Reynolds number turbulent channel flow is possible. Scale separation at high Reynolds numbers disclosed extended log layers (Smits et al., 2011) and very long streamwise organized motions of the order of tens of boundary layer thicknesses. These are variously named as very large-scale motions (VSLM) (Kim \& Adrian, 1999), superstruc55 tures (Hutchins \& Marusic, 2007b), global modes (del Álamo \& Jiménez, 2003. 
Hoyas \& Jimnez, 2006), or global structures (del Álamo \& Jiménez, 2006) and have been observed in pipes (Kim \& Adrian, 1999, Guala et al., 2006), channel flows (Jiménez, 1998, Liu et al., 2001; del Álamo \& Jiménez, 2003, Hoyas \& Jimnez, 2006) and spatially developing boundary layers (Balakumar \& Adrian, 60 2007, Hutchins \& Marusic, 2007b, Lee \& Sung, 2011). Although the genesis and formation of large scale motions are not exactly known, Kim \& Adrian (1999) conjectured that they are streamwise-aligned hairpin vortex packets. Jiménez (1998) suggested that the formation of these structures can be ascribed to the combined linear effect of impermeability of the wall and the existence of the mean shear. It has been confirmed that LSM contain most of the kinetic energy Jiménez (1998); Guala et al. (2006) as well as Reynolds stress (Guala et al. 2006). In fact, Guala et al. (2006) found that VLSM detected in fully developed pipe flows carry more than $50 \%$ of the Reynolds stresses. Liu et al. (2001) extracted large-scale modes in a turbulent channel using proper orthogonal decomposition (POD) and concluded that those modes contain half of the total kinetic energy and $65-75 \%$ of the total Reynolds stress. Hence, the dynamical significance of LSM in the logarithmic layer is evident. Besides these structures are the dominant feature in the logarithmic and wake regions of the boundary layer (Liu et al., 2001; Hutchins \& Marusic, 2007a), their effects on small-scale 75 near-wall turbulence has also been studied (Hutchins \& Marusic, 2007a). In fact, Mathis et al. (2009) reported that near-wall small scale motions are amplitude modulated by the existence of LSM in the logarithmic layer.

Although it was noted that LSM promote scalar transport in turbulent flows (Tennekes \& Lumley, 1972), the topology of the LSM which contribute to the scalar transport in comparison with LSM which are significant in transporting momentum have not been investigated. Hence, the present study utilizes a DNS database of a turbulent channel flow at $R e_{\tau}=395$ to investigate whether the physical structure of LSM which are responsible for transporting most of the turbulent kinetic energy and Reynolds stresses is similar to the structure of LSM which are carrying scalar fluxes.

This manuscript is organized as follows: a brief description of numerical 
procedure and comparison of mean and second order statistics are shown in $\S 2$ $\S 3$ elaborates results of the three-dimensional two-point correlations. Finally, conclusions are laid out in $\S 4$.

\section{Numerical procedure}

Continuity, momentum and passive scalar transport equations are shown in their non-dimensional form in equations (1), (2), and (3). Non-dimensional form is derived using outer coordinates: channel centerline velocity, $U_{c}$, channel half height, $h$, upper cold wall temperature, $T_{W C}$, and lower hot wall temperature $T_{W H}$. Non-dimensional temporal and spatial coordinates are defined as $t=$ $t^{*} U_{c} / h$ and $x_{i}=x_{i}^{*} / h$ respectively, where, superscript $*$ denotes dimensional values. Dimensionless temperature is prescribed as $\theta=1-2\left[\left(T_{W H}-T^{*}\right) /\left(T_{W H}-T_{W C}\right)\right]$ while non-dimensional pressure is defined by $p=p^{*} / \rho U_{c}^{2}$. Thus, following nondimensional governing equations are derived.

$$
\begin{gathered}
\frac{\partial u_{i}}{\partial x_{i}}=0 \\
\frac{\partial u_{i}}{\partial t}+u_{j} \frac{\partial u_{i}}{\partial x_{j}}=-\frac{1}{\rho} \frac{\partial p}{\partial x_{i}}+\frac{1}{R e_{h}} \frac{\partial^{2} u_{i}}{\partial x_{j} \partial x_{j}}+\Pi \\
\frac{\partial \theta}{\partial t}+u_{j} \frac{\partial \theta}{\partial x_{j}}=\frac{1}{R e_{h} \operatorname{Pr}} \frac{\partial^{2} \theta}{\partial x_{j} \partial x_{j}}
\end{gathered}
$$

where, $\Pi$ is the constant pressure gradient which provides the driving force to maintain the constant flow rate through the channel. $R e_{h}=U_{c} h / \nu$ is the Reynolds number based on outer scales. $U_{c}$ is the laminar Poiseuille velocity at the centerline and assumed unity for convenience. $\operatorname{Pr}=\nu / \alpha$ is the molecular Prandtl number where $\nu$ and $\alpha$ are molecular diffusivities of momentum (or kinematic viscosity) and scalar respectively . Governing equations are discretized using a staggered central second order finite difference scheme in an orthogonal coordinate system. The scheme is based on the fractional step factorization method (Kim \& Moin, 1985). Time advancing of linear terms is done by using implicit (Crank-Nicholson) method while an explicit ( $3^{\text {rd }}$ order Runge Kutta) 


\begin{tabular}{lllllllll}
\hline \hline$R e_{\tau}$ & $L_{x} / h$ & $L_{z} / h$ & $L_{y} / h$ & $\Delta x^{+}$ & $\Delta z^{+}$ & $\Delta y_{\max }^{+}$ & $\Delta y_{\min }^{+}$ & $N_{x}, N_{y}, N_{z}$ \\
\hline 395 & $4 \pi$ & $\pi$ & 2 & 8.6 & 6.4 & 11.6 & 0.095 & $577,193,193$ \\
\hline \hline
\end{tabular}

Table 1: Parameters of the simulations. the molecular Prandtl number is lower than one (i.e., $\operatorname{Pr}=0.71$ ); thus, the Obukhoff-Corrsin thermal length scale is defined as $\eta_{c}=\eta_{k} P r^{(-3 / 4)}$. Hence, Obukhoff-Corrsin length scales are approximately 30\% larger than the Kol- 
mogorov length scales. This ensures the proper resolution of the all the scales of the thermal field by capturing Kolmogorov length scales.

\subsection{Mean and second order statistics}

(a)

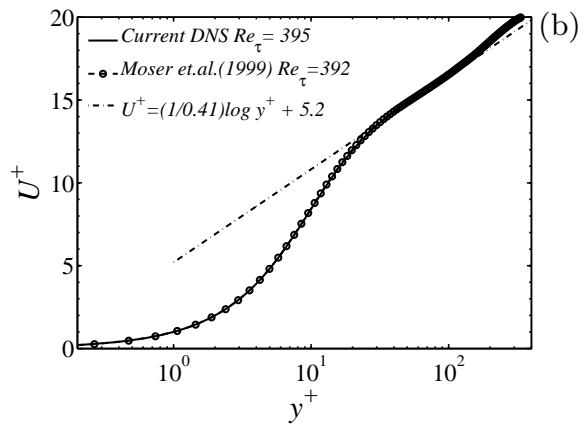

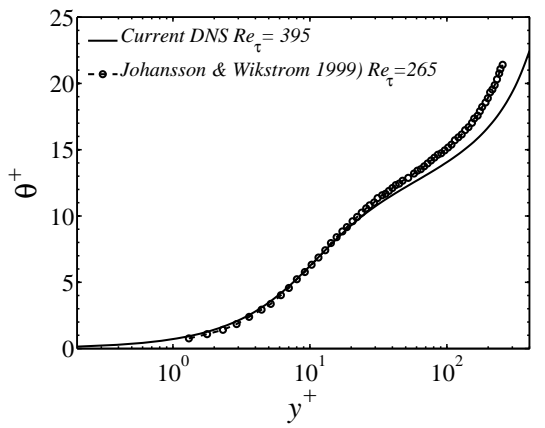

Figure 1: Mean profiles of (a) streamwise velocity and (b) scalar field in wall coordinates.

Mean profiles of both streamwise velocity and scalar are shown and compared with other DNS data in figures $1 \mathrm{a}$ and $1 \mathrm{~b}$ respectively. The mean velocity profile nearly overlaps the Moser et al. (1999) data. The deviation detected in mean scalar profile can be ascribed to the difference in Reynolds numbers in current simulation and Johansson \& Wikström (1999) data at $R e_{\tau}=265$.

(a)

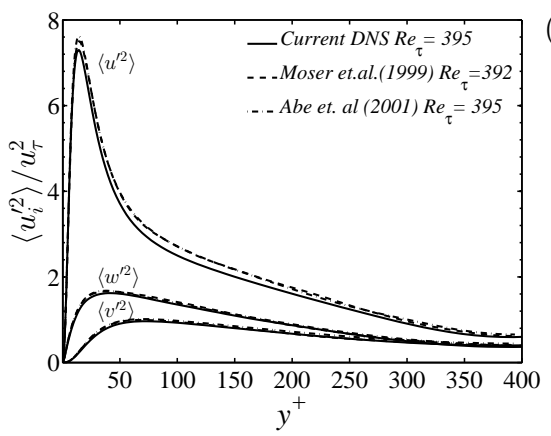

(b)

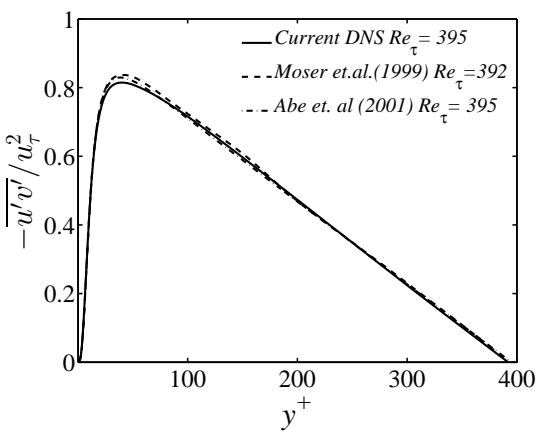

Figure 2: Profiles of (a) Reynolds normal stresses and (b) Reynolds shear stress. All variables are normalized in wall units.

Second order statistics for the velocity field namely Reynolds normal stresses and wall-normal Reynolds shear stress were compared with DNS data from 
Moser et al. (1999) and Abe et al. (2001) at similar friction Reynolds numbers. Present data shows excellent agreement specially in the near wall region.The slight deviation which is seen in outer layers is insignificant (less than 5\%).

(a)

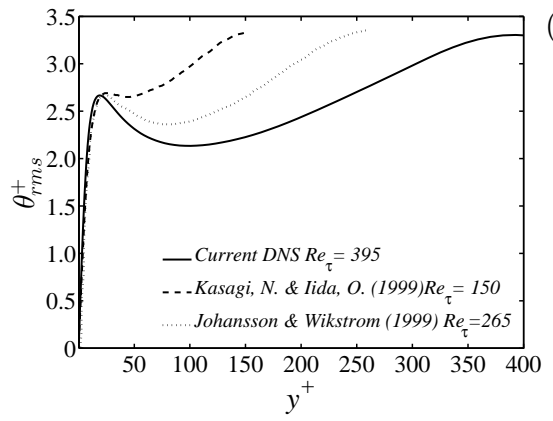

(b)

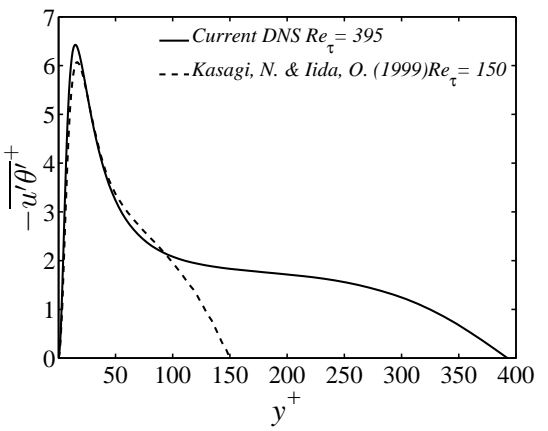

(c)

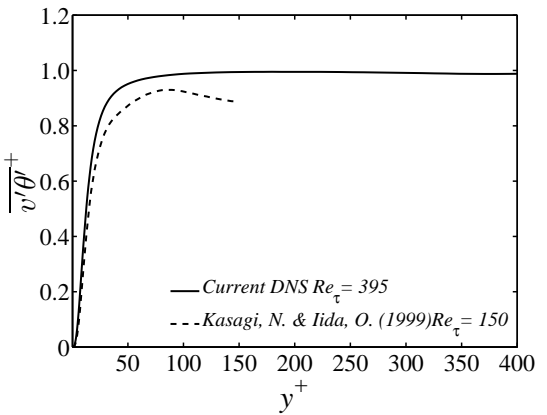

Figure 3: Profiles of (a) $\theta_{r m s}^{+}$, (b) streamwise heat flux, and (c) wall-normal heat flux profiles. All variables are normalized in wall units.

Standard deviation of scalar fluctuation, streamwise heat flux, and wallnormal heat flux are shown in figures $3 \mathrm{a}, 3 \mathrm{~b}$, and $3 \mathrm{c}$ respectively. DNS data of Johansson \& Wikström (1999) and Kasagi \& Iida (1999) are used for the comparison. However, the Reynolds numbers of those experiments are lower than that of the current numerical experiment. The $\theta_{r m s}^{+}$profile shows a very good agreement with the DNS data from Johansson \& Wikström (1999) and Kasagi \& Iida (1999) in the near wall region although a deviation is detected in the log layer and outer layer. This is mainly due to the difference in Reynolds number between the numerical experiments. Data collapse in the outer region when normalized in outer units (not shown here) confirms the above claim. 
Scalar fluctuations show a maximum near the centerline due to the thermal production owing to the gradient of the mean scalar field. The local maximum around $y^{+}=20$ is caused by steep gradients of the mean scalar field which provokes high thermal production in the buffer region. Correspondingly, the profile of streamwise fluctuation also peaks near $y^{+}=20$. This is ascertainable by noticing analogous peaks in both streamwise velocity (see figure 2a) variance and standard deviation of scalar fluctuations (see figure 3a). Which is consistent with the high level of correlation between $u^{\prime}$ and $\theta^{\prime}$ in the buffer layer, according to figure $3 \mathrm{~b}$ for the streamwise heat fluxes. It is interesting to observe a plateau in the wall-normal heat flux profile beyond $y^{+}=50$ after a steep climb from the wall. Teitel \& Antonia (1993) and Araya et al. (2008) stated that at very high Reynolds numbers scalar transport equation in fully developed channel flows 170 with similar boundary conditions as in this study can be simplified into

$$
\frac{1}{\operatorname{Pr}} \frac{\partial \Theta^{+}}{\partial y^{+}}-\overline{v^{\prime+} \theta^{\prime+}}=-1
$$

from which it can be deduced that wall-normal turbulent scalar flux depends on the molecular Prandtl number and the mean scalar gradient. Mean scalar gradient in the current study, decreases as the center of the channel is reached (see figure 1b). This makes wall-normal scalar flux to become a constant at the channel center. Since, the equation 4 is only valid when $R e \rightarrow \infty$, slight deviation from the uniform value is observed at lower $R e$ numbers.

\section{Two-point correlations}

Two-point cross-correlations are computed in order to identify large scale motions that carry significant amounts of turbulent kinetic energy, Reynolds stresses, scalar variances and scalar fluxes. The cross-correlations and autocorrelations are computed in Fourier space and then inverse transformed to the physical space whenever required. This significantly reduces the number of computations. Cross-correlation $\left(\rho_{\alpha^{\prime} \beta^{\prime}}\right)$ of two arbitrary mean zero variables $\alpha^{\prime}$ 
and $\beta^{\prime}$ for a turbulent channel flow is defined as,

$$
\rho_{\alpha^{\prime} \beta^{\prime}}\left(\delta x, y_{0}, y, \delta z\right)=\frac{R_{\alpha^{\prime} \beta^{\prime}}\left(\delta x, y_{0}, y, \delta z\right)}{\alpha_{r m s}^{\prime} \beta_{r m s}^{\prime}} .
$$

Cross covariance of the same random variables $\alpha^{\prime}$ and $\beta^{\prime}, R_{\alpha^{\prime} \beta^{\prime}}$, is the inverse Fourier transform of $\hat{R}_{\alpha^{\prime} \beta^{\prime}}$ given by,

$$
\hat{R}_{\alpha^{\prime} \beta^{\prime}}\left(k_{x}, y_{0}, y, k_{z}\right)=\left\langle\hat{\alpha}^{\prime}\left(k_{x}, y, k z\right) \hat{\beta}^{{ }^{*}}\left(k_{x}, y_{0}, k z\right)\right\rangle .
$$

In equation (6), $\hat{\alpha}^{\prime}$ is the Fourier transform of $\alpha^{\prime}$ and \langle\rangle denotes the time average. $\hat{\alpha}^{\prime *}$ is the complex conjugate of the variable. The reference location at which the computation is based upon is denoted by $\left(x_{0}, y_{0}, z_{0}\right)$ while $(x, y, z)$ represents (1) any arbitrary location in the domain. This follows that $\delta x=x-x_{0}$ and $\delta z=z-z_{0}$. $k_{x}$ and $k_{z}$ are streamwise and spanwise wavenumbers respectively. Note that $x$ and $z$ are the homogeneous directions. Thus, $R_{\alpha^{\prime} \beta^{\prime}}$ in equation (5) becomes a function of separations $\delta x$ and $\delta z$ in $x$ and $z$ directions respectively. This definition of cross-correlation has been used by Sillero et al. (2014) in both turbulent channels and boundary layers which is statistically well-defined and asymptotically unbiased. Spatial averaging of covariances was not carried out to avoid the destruction of structures in any of the homogeneous directions. Although correlations are computed adjacent to the both wall, structures which are close to the lower wall only presented in this manuscript.

\section{1. $3 D$ representation of correlations}

Figures 4 5, and 6illustrate three dimensional representation of $\rho_{u^{\prime} u^{\prime}}, \rho_{v^{\prime} v^{\prime}}$, $\rho_{\theta^{\prime} \theta^{\prime}}, \rho_{u^{\prime} v^{\prime}}, \rho_{u^{\prime} \theta^{\prime}}$, and $\rho_{v^{\prime} \theta^{\prime}}$. Iso-surfaces of $\rho_{u^{\prime} u^{\prime}}, \rho_{v^{\prime} v^{\prime}}, \rho_{\theta^{\prime} \theta^{\prime}}, \rho_{u^{\prime} v^{\prime}}, \rho_{u^{\prime} \theta^{\prime}}$, and $\rho_{v^{\prime} \theta^{\prime}}$ depict the physical extent of the LSM based on three regions: buffer region $\left(y_{0}^{+} \approx 20\right), \log$ region $\left(y_{0}^{+} \approx 69\right)$, and wake region $\left(y_{0}^{+} \approx 170\right)$. This helps the visualization of the evolution of structures with respect to the distance from the wall. Iso-surfaces are shown at the iso-value of 0.2 for $\rho_{u^{\prime} u^{\prime}}, \rho_{v^{\prime} v^{\prime}}, \rho_{\theta^{\prime} \theta^{\prime}}$, and $\rho_{v^{\prime} \theta^{\prime}}$ where the variables are positively correlated. When variables are negatively correlated, such as in $\rho_{u^{\prime} v^{\prime}}$ and $\rho_{u^{\prime} \theta^{\prime}}$, the iso-surfaces are set to -0.2 . Since it is well known that the large scale turbulent motions in shear flows are highly 
anisotropic, the analysis of three dimensional two-point correlations provides an accurate representation of LSM.

(a)

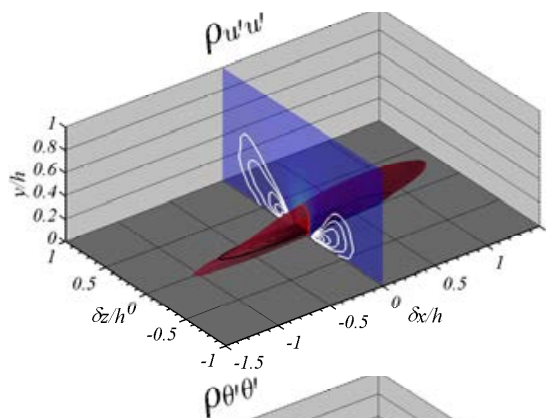

(c)

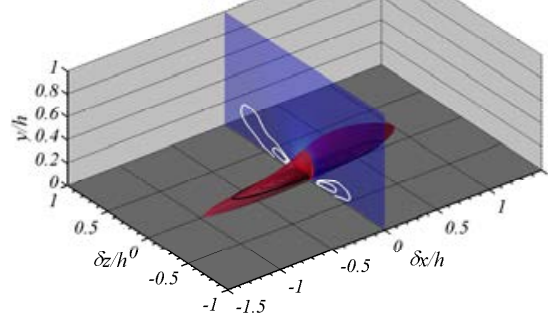

(e)

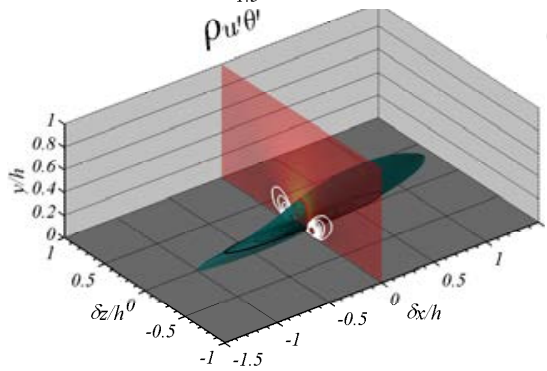

(b)

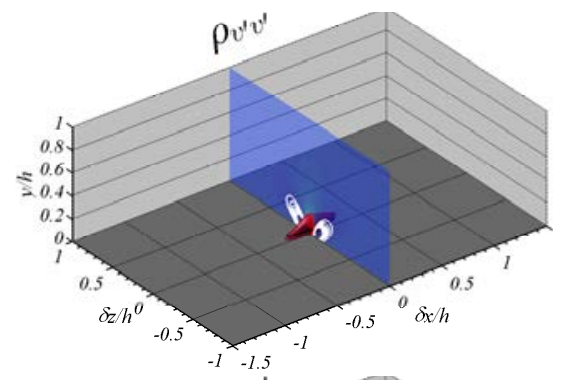

(d)

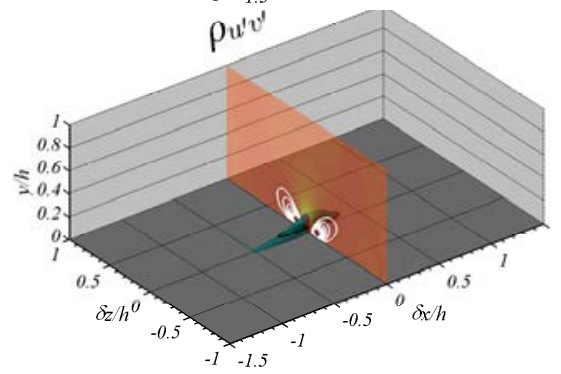

(f)

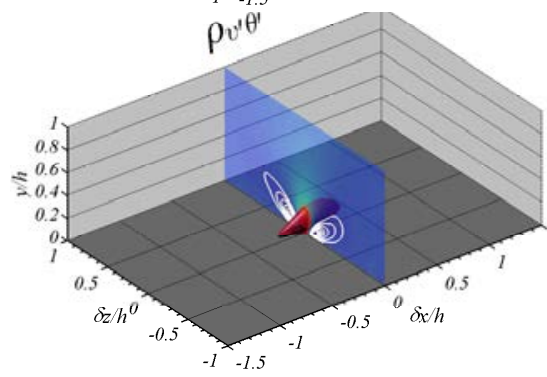

Figure 4: Three dimensional view of (a) $\rho_{u^{\prime} u^{\prime}}$ (b) $\rho_{v^{\prime} v^{\prime}}$ (c) $\rho_{\theta^{\prime} \theta^{\prime}}$ (d) $\rho_{u^{\prime} v^{\prime}}$ (e) $\rho_{u^{\prime} \theta^{\prime}}$ correlations are computed with respect to the wall-normal location $y_{0}^{+} \approx 20\left(y_{0} / h \approx 0.054\right)$. Iso-surfaces are drawn at $\rho_{\alpha^{\prime} \beta^{\prime}}=0.2$ (crimson) if the positive correlation of variables is dominant and at $\rho_{\alpha^{\prime} \beta^{\prime}}=-0.2$ (dark cyan) if the negative correlation of variables is dominant.

White lines drawn on the vertical cut plane at $\delta x / h=0$ of figures $4 \mathrm{a}, 4 \mathrm{~b}, 4 \mathrm{c}$ and $4 \mathrm{f}$ show negatively correlated contours from -0.09 with increments of 0.01 . The same lines in figures $4 \mathrm{~d}$ and $4 \mathrm{e}$ represents positively correlated contours from 0.02 with increments of 0.01 . The structure of $\rho_{u^{\prime} u^{\prime}}$ depicts the longest streamwise elongation. Fluctuations of streamwise velocity $\left(u^{\prime}\right)$ are created by the perturbed packets of fluid travel back and forth from one region to another 
with a different level of momentum. The driving force behind these packets is the wall normal velocity fluctuations $\left(v^{\prime}\right)$. Orlandi \& Jiménez (1994) stated that streamwise fluctuations behave in the same manner that a passive scalar is transported by the transverse velocity fluctuations. Fully developed turbulent channel has similar mean velocity profiles at all the streamwise locations. Thus, fluid particles which are transported from the similar wall-normal regions have approximately the same values of streamwise velocity fluctuations. This makes streamwise component of fluctuations significantly correlated in the streamwise direction. On the contrary, mean value of wall-normal velocity is vanishingly small across the boundary layer. Hence, the wall-normal velocity fluctuations of fluid particles are not as high as their streamwise counterpart. This causes $\rho_{v^{\prime} v^{\prime}}$ to die out faster resulting in shorter streamwise extensions as shown in figure $4 \mathrm{~b}$ 230 A similar characteristics can be observed in spanwise velocity fluctuations as well (not shown here). The size and the shape of $\rho_{\theta^{\prime} \theta^{\prime}}$ and $\rho_{u^{\prime} \theta^{\prime}}$ closely resemble those of $\rho_{u^{\prime} u^{\prime}}$ at the near-wall region (i.e., at $y_{0}^{+} \approx 20$ ). This similarity can be ascribed to the similarity of both the physical mechanism and the mean scalar profile that generate comparable levels of fluctuations only at the near wall region by the streamwise velocity field. Also notice the peak values of $\overline{u^{\prime 2}}, \theta_{r m s}^{+}$, and $\overline{u^{\prime+} \theta^{\prime+}}$ pccur around $y^{+}=20$ in figures 2a, 3a, and 3b respectively. Moreover, Kim \& Moin (1987) also found a strong correlation between $u^{\prime}$ and $\theta^{\prime}$. This is consistent with the present observations in the near-wall region. Slight differences of $\rho_{\theta^{\prime} \theta^{\prime}}$ and $\rho_{u^{\prime} \theta^{\prime}}$ could be related to differences in molecular diffusion of the momentum and the scalar and the presence of a small favorable pressure gradient in the turbulent channel. According to Kong et al. (2001), the pressure gradient is a source of dissimilarity between the velocity and the thermal field. The close affinity in the angle inclination of $\rho_{u^{\prime} u^{\prime}}, \rho_{\theta^{\prime} \theta^{\prime}}$, and $\rho_{u^{\prime} \theta^{\prime}}$ which is observed only in the near wall region confirms the similarity of structures that contribute most of the streamwise component of turbulent kinetic energy, scalar variance, and streamwise scalar flux. One can observe that the iso-surface of $\rho_{v^{\prime} \theta^{\prime}}$ resembles that of $\rho_{v^{\prime} v^{\prime}}$. However, the slight change in wall-normal dimension of $\rho_{v^{\prime} \theta^{\prime}}$ could be caused by the disparity in molecular diffusivities of the scalar and 
the momentum and the deviation of turbulent Prandtl number $\left(P r_{t}\right)$ from the unity. The structure of $\rho_{u^{\prime} v^{\prime}}$ is considerably smaller than that of $\rho_{u^{\prime} u^{\prime}}, \rho_{u^{\prime} \theta^{\prime}}$, and $\rho_{\theta^{\prime} \theta^{\prime}}$. This corresponds to the relatively lower levels of wall-normal velocity fluctuations. Notice that the variance of wall-normal velocity fluctuations has to lowest values in figure $2 \mathrm{a}$

(a)

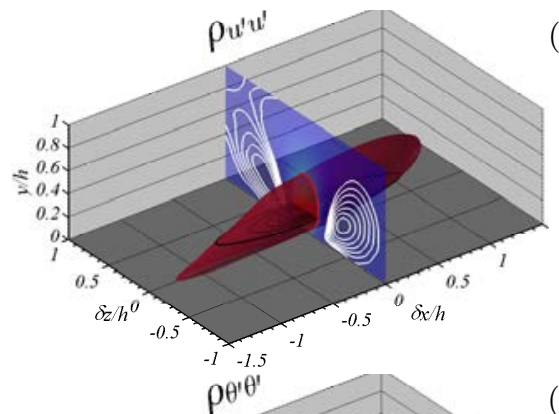

(c)

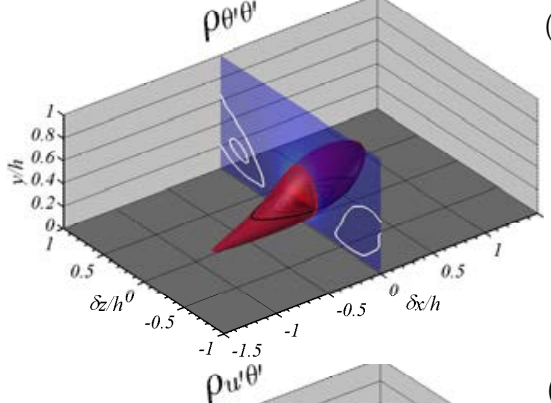

(e)

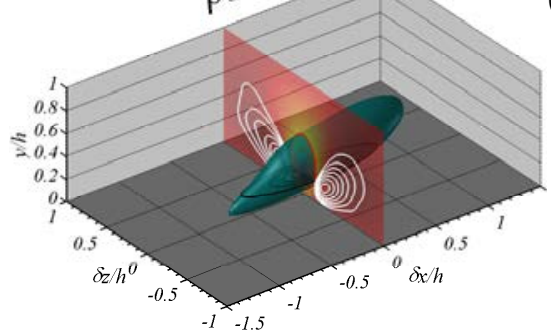

(b)

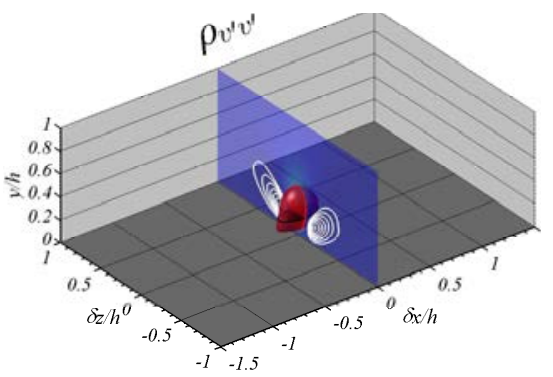

(d)

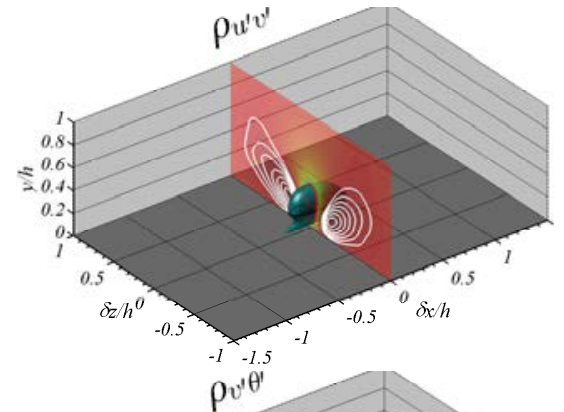

(f)

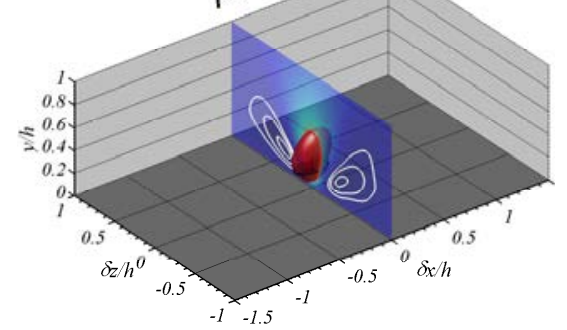

Figure 5: Three dimensional view of (a) $\rho_{u^{\prime} u^{\prime}}$ (b) $\rho_{v^{\prime} v^{\prime}}$ (c) $\rho_{\theta^{\prime} \theta^{\prime}}$ (d) $\rho_{u^{\prime} v^{\prime}}$ (e) $\rho_{u^{\prime} \theta^{\prime}}$ correlations are computed with respect to the wall-normal location $y_{0}^{+} \approx 69\left(y_{0} / h \approx 0.18\right)$. Iso-surfaces are drawn at $\rho_{\alpha^{\prime} \beta^{\prime}}=0.2$ (crimson) for positively correlated variables and at $\rho_{\alpha^{\prime} \beta^{\prime}}=-0.2$ (dark cyan) for negatively correlated variables.

It is clearly seen in figure 5 (at $y^{+} \approx 69$ ), that particularly the spanwise and 255 wall-normal extents of all the correlations grow in the log layer. This means that LSM primarily reside in the log layer. Wall-normal fluctuations are less 
restricted away from the wall since the blocking effect of the wall possesses less influence on $v^{\prime}$. This promotes structures to grow in the wall-normal direction creating $y$-elongated (thick) structures.It is noticeable that the angles of inclination of $\rho_{u^{\prime} u^{\prime}}$ and $\rho_{u^{\prime} \theta^{\prime}}$ remain more or less the same as in the buffer layer.

(a)

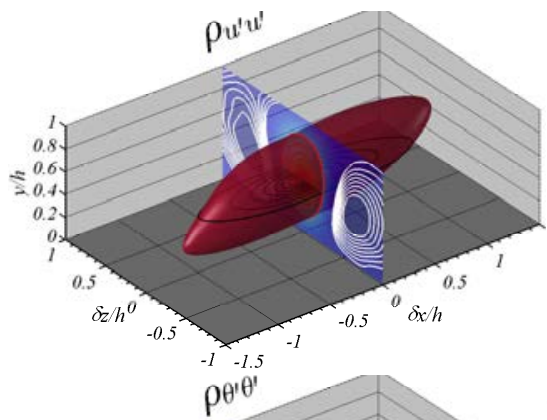

(c)

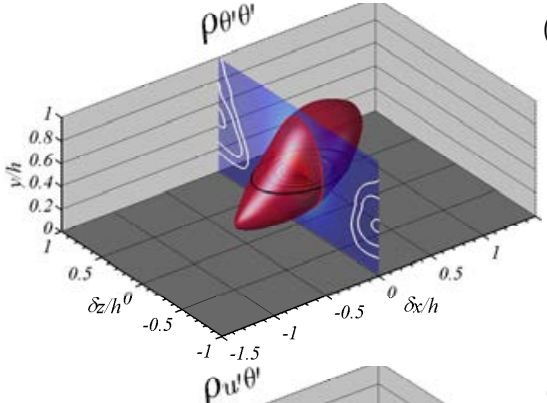

(e)

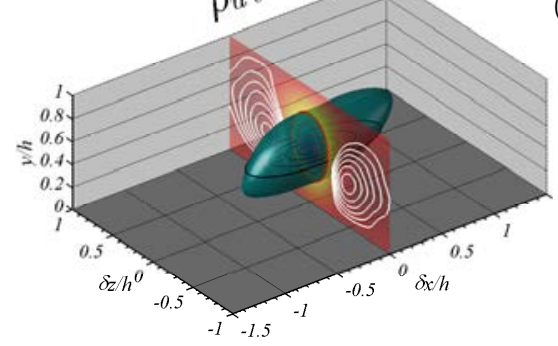

(b)

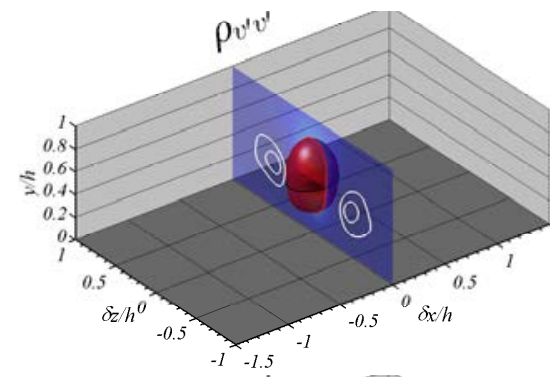

(d)

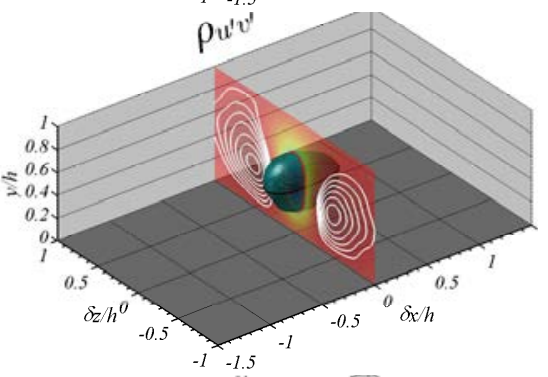

(f)

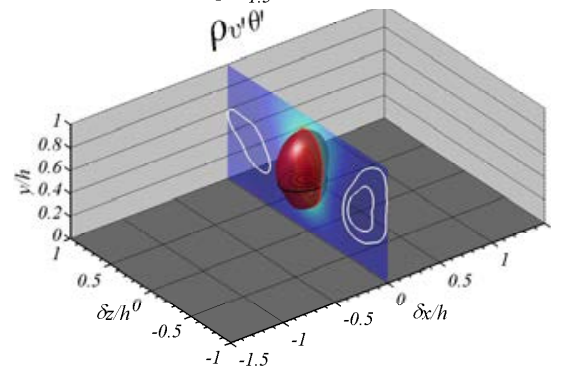

Figure 6: Three dimensional view of (a) $\rho_{u^{\prime} u^{\prime}}$ (b) $\rho_{v^{\prime} v^{\prime}}$ (c) $\rho_{\theta^{\prime} \theta^{\prime}}$ (d) $\rho_{u^{\prime} v^{\prime}}$ (e) $\rho_{u^{\prime} \theta^{\prime}}$ correlations are computed with respect to the wall-normal location $y_{0}^{+} \approx 170\left(y_{0} / h \approx 0.45\right)$. Iso-surfaces are drawn at $\rho_{\alpha^{\prime} \beta^{\prime}}=0.2$ (crimson) for positively correlated variables and at $\rho_{\alpha^{\prime} \beta^{\prime}}=-0.2$ (dark cyan) for negatively correlated variables.

However, the angle of inclination of $\rho_{\theta^{\prime} \theta^{\prime}}$ has been increased. Streamwise extent of all but $\rho_{u^{\prime} v^{\prime}}$ structures remain approximately constant in both regions of the boundary layer. Small structures in $\rho_{v^{\prime} v^{\prime}}, \rho_{u^{\prime} v^{\prime}}$, and $\rho_{v^{\prime} \theta^{\prime}}$ are consequences of small correlations in the wall-normal velocity, thus structures are elongated 
field. Notice that the structure of $\rho_{u^{\prime} u^{\prime}}$ is similar to that of $\rho_{u^{\prime} \theta^{\prime}}$. The same can be noted for the structure of $\rho_{v^{\prime} v^{\prime}}$ and $\rho_{v^{\prime} \theta^{\prime}}$. Although the wall-normal extent of the $\rho_{u^{\prime} v^{\prime}}$ structure and that of $\rho_{v^{\prime} \theta^{\prime}}$ relatively in the same limits, notable disparity of their streamwise lengths makes them different.

270 wake region (i.e., at $y_{0}^{+} \approx 170$ ) are shown in figure 6 . Notice the streamwise and spanwise lengh scales of $\rho_{u^{\prime} u^{\prime}}$ structure are around $2-3 h$ and $1 h$ repectively. This is consistent with the dimensions of the structures observed by Kovasznay et al. (1970). Hence, we effectively capture the largest scales at the outer region of dimensions is evident. This is produced by the increased fluctuations of wallnormal velocity. It is clear that the structural resemblance of $\rho_{u^{\prime} u^{\prime}}$ and $\rho_{\theta^{\prime} \theta^{\prime}}$ which is noticeable in the buffer region has completely been destroyed. This is attributed to the centerline peak of $\theta_{r m s}^{+}$provoked by a significant thermal region. It implies clearly distinct formation of $u^{\prime}$-structures and $\theta^{\prime}$-structures. However, the morphological analogy between $\rho_{u^{\prime} u^{\prime}}$ and $\rho_{u^{\prime} \theta^{\prime}}$ seems to be intact, although minor difference in streamwise lengths are detected.

The structural similarity between $\rho_{u^{\prime} v^{\prime}}$ and $\rho_{v^{\prime} \theta^{\prime}}$ is more pronounced than that between $\rho_{u^{\prime} v^{\prime}}$ and of $\rho_{u^{\prime} \theta^{\prime}}$ as observed in figures 6d, 6e, and 6f This coincides with the observation established by Perry \& Hoffmann (1976) in which they detected high correlation between the events that contribute to most of the Reynolds stress and wall-normal heat fluxes at the outer layer of a turbulent boundary layer with uniform temperature boundary. Inclination of both $\rho_{u^{\prime} u^{\prime}}$ and $\rho_{u^{\prime} \theta^{\prime}}$ has been reduced from the value that they showed in the buffer layer and in the log layer. However, the angle of inclination of $\rho_{\theta^{\prime} \theta^{\prime}}$ has increased significantly. This points out the structural differences in eddies that contribute most of the scalar variance and streamwise kinetic energy in the outer layer. The phenomena seems to be quiet different in the near-wall region $\left(y^{+} \leq 30\right)$ of the boundary layer as observed in figure 4. Two dimensional cross sectional 
views on three mutually perpendicular directions are examined to obtain further insight into the foregoing observations.

\subsection{2 dimensional veiws of correlations}

(a)

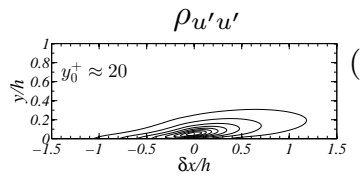

(d)

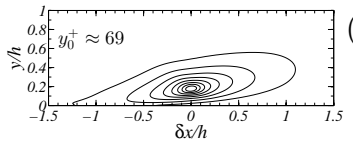

(g)

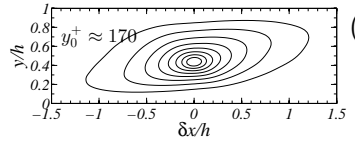

(b)

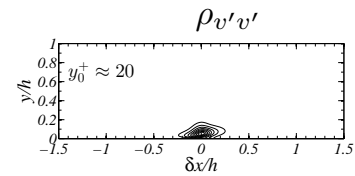

(e)

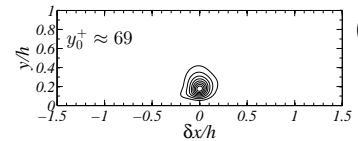

(h)

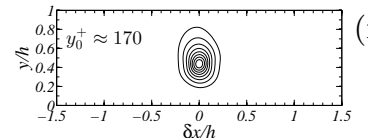

(c)

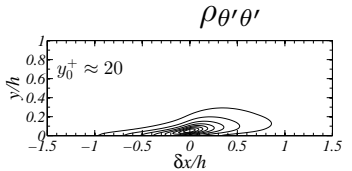

(f)

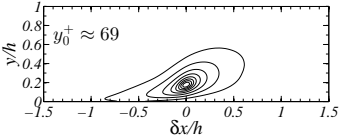

(i)

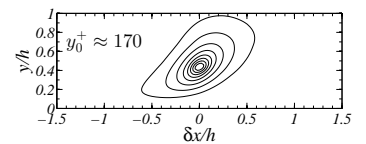

Figure 7: Two-dimensional sections of $\rho_{u^{\prime} u^{\prime}}$ in (a) (d) and (g); of $\rho_{v^{\prime} v^{\prime}}$ in (b) (e) and (h) ; of $\rho_{\theta^{\prime} \theta^{\prime}}$ in (c) (f) and (i) on streamwise wall-normal plane. Reference point is located at: $y_{0} / h \approx 0.054\left(y_{0}^{+} \approx 20\right)$ for $(\mathrm{a})(\mathrm{c}) y_{0} / h \approx 0.18\left(y_{0}^{+} \approx 69\right)$ for $(\mathrm{d}) \quad(\mathrm{f})$ and $y_{0} / h \approx 0.45\left(y_{0}^{+} \approx 170\right)$ for (g) (i) Contour levels are from 0.2 from 0.9 with the increment of 0.1

In figure 7, two dimensional sections of $\rho_{u^{\prime} u^{\prime}}, \rho_{v^{\prime} v^{\prime}}$, and $\rho_{\theta^{\prime} \theta^{\prime}}$ on the streamwise wall-normal plane are shown. The difference in angles of inclination between $\rho_{u^{\prime} u^{\prime}}$ and that of $\rho_{\theta^{\prime} \theta^{\prime}}$ is apparent particularly in figures $7 \mathrm{~d}, 7 \mathrm{f}, 7 \mathrm{~g}$, and 7i. It is evident that there is no streamwise inclination involved in the structure of $\rho_{v^{\prime} v^{\prime}}$. In particular, notice the change in angle of inclination of $\rho_{u^{\prime} u^{\prime}}$ and $\rho_{\theta^{\prime} \theta^{\prime}}$. The structure of $\rho_{\theta^{\prime} \theta^{\prime}}$ steepen when the reference point moves away from the wall while the angle of inclination of $\rho_{u^{\prime} u^{\prime}}$ structure remains approximately the same. Antonia et al. (2009) also detected a similar disparity in the angles of inclination of $\rho_{u^{\prime} u^{\prime}}$ and $\rho_{\theta^{\prime} \theta^{\prime}}$ at the outer layer $(y / h \approx 0.5)$ in a turbulent channel at similar $R e_{\tau}$. Their study, however, did not comment on the inclination angles at the near wall regions. Streamwise lengths of $\rho_{u^{\prime} u^{\prime}}$ and $\rho_{v^{\prime} v^{\prime}}$ are more or less constant at all the levels of the boundary layer. In contrary, streamwise length of $\rho_{\theta^{\prime} \theta^{\prime}}$ structure changes approximately form $1.8 h$ to $1.2 h$ when it moves away from the wall. A superficial observation may conclude that 
this is an outcome of passive motion of scalar particles with streawise velocity fluctuations in the buffer region and with wall-normal velocity fluctuations in the outer layer. Nevertheless, with much scrutiny this difference can be ascribed to the concentration or attraction of scalars to a topologically distinct structure than one that contribute most of the kinetic energy especially in the outer layer of the boundary layer.

(a)
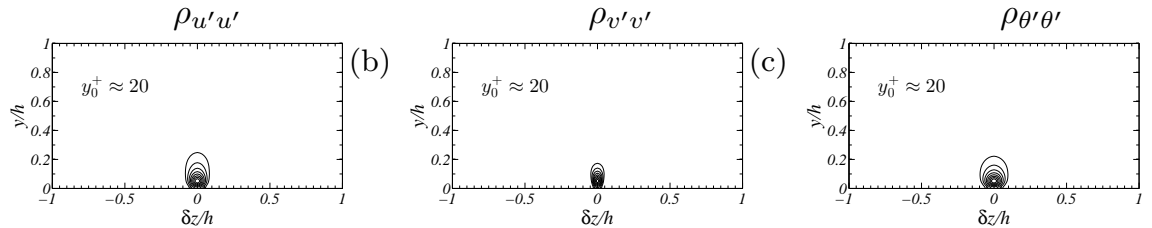

(d)
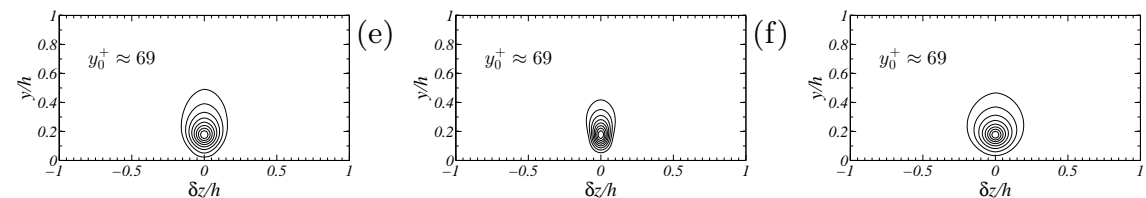

(g)
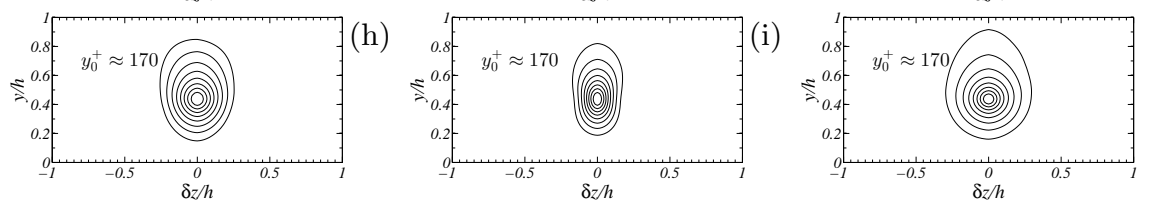

Figure 8: Two-dimensional sections of $\rho_{u^{\prime} u^{\prime}}$ in (a) (d) and (g); of $\rho_{v^{\prime} v^{\prime}}$ in (b) (e) and (h) ; of $\rho_{\theta^{\prime} \theta^{\prime}}$ in (c) (f) and (i) on spanwise wall-normal plane. Reference point is located at : $y_{0} / h \approx 0.054\left(y_{0}^{+} \approx 20\right)$ for $(\mathrm{a})(\mathrm{c}) y_{0} / h \approx 0.18\left(y_{0}^{+} \approx 69\right)$ for $(\mathrm{d})(\mathrm{f})$ and $y_{0} / h \approx 0.45\left(y_{0}^{+} \approx 170\right)$ for (g) (i) Contour levels are from 0.2 from 0.9 with the increment of 0.1

Two dimensional view on spanwise wall-normal plane shown in figure 8 renders an accurate picture of geometrical changes of correlations along the spanwise direction. In general, all the structures expand along the spanwise direction as the outer layer is reached. It is worth to notice the close resemblance of spanwise dimensions of $\rho_{u^{\prime} u^{\prime}}$ and $\rho_{\theta^{\prime} \theta^{\prime}}$ regardless of the reference location. This implies that streamwise velocity fluctuation passively moved not only by wall-normal perturbations but also by spanwise perturbations. This was first detected by Lyons et al. (1991) through their two-point spatial auto-correlations maps of $u^{\prime}$ and $\theta^{\prime}$ on spanwise wall-normal plane. However, their observation was only limited to the buffer region and the number of flow fields considered for 
the ensemble seems to be insufficient. The present study provides a comprehensive analysis since it probes complete three dimensional picture of correlations and thus, all the structure of both momentum and thermal fields. Correspondingly, Antonia et al. (2009) found a remarkable spanwise spectral resemblance between $u^{\prime}$ and $\theta^{\prime}$. Additionally, a careful examination of $8 \mathrm{a}$ and $8 \mathrm{c}$ reveals that the spanwise extent of the $\rho_{u^{\prime} u^{\prime}}$ and $\rho_{\theta^{\prime} \theta^{\prime}}$ structures are approximately $100 \nu / u_{\tau}$.

This is in compliance with the generally accepted value of velocity and thermal streaks detected in the near-wall region (Iritani et al., 1985; Kong et al. 2000 ).

(a)

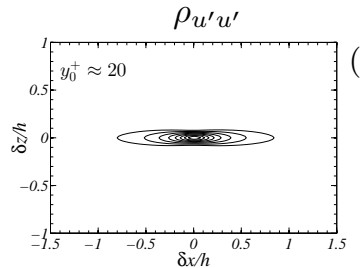

(d)

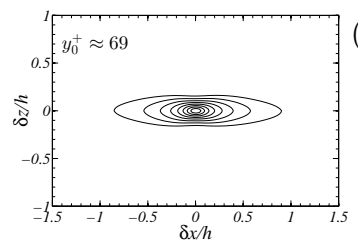

(g)

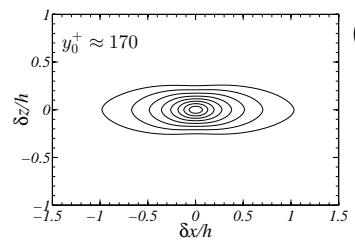

(b)

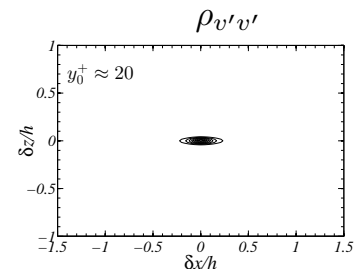

(e)

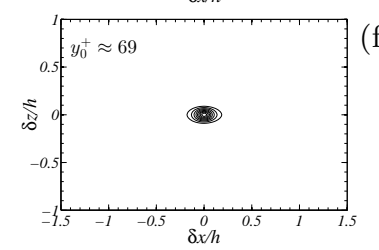

(h)

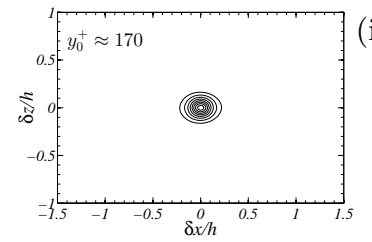

(c)

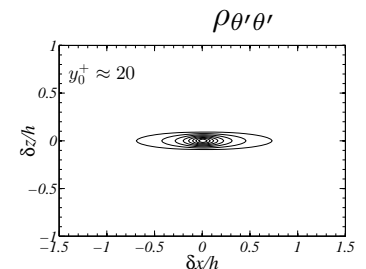

(f)

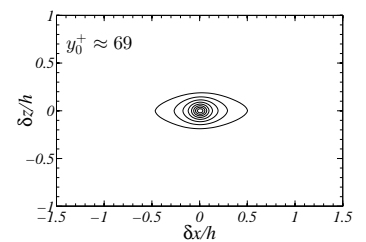

(i)

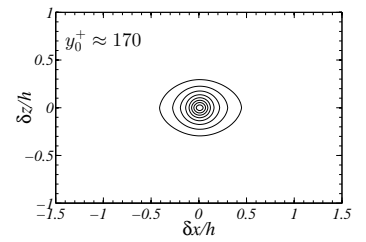

Figure 9: Two-dimensional sections of $\rho_{u^{\prime} u^{\prime}}$ in (a) (d) and (g); of $\rho_{v^{\prime} v^{\prime}}$ in (b) (e), and (h) ; of $\rho_{\theta^{\prime} \theta^{\prime}}$ in (c) [f) and (i) on spanwise streamwise plane. Reference point is located at: $y_{0} / h \approx 0.054\left(y_{0}^{+} \approx 20\right)$ for $(\mathrm{a})[\mathrm{cc}) y_{0} / h \approx 0.18\left(y_{0}^{+} \approx 69\right)$ for $(\mathrm{d})[\mathrm{f})$ and $y_{0} / h \approx 0.45\left(y_{0}^{+} \approx 170\right)$ for $(\mathrm{g})$ (i) Contour levels are from 0.2 from 0.9 with the increment of 0.1

Figure 9 shows sectional view of correlations on the spanwise streamwise plane. Streamwise lengths of both $\rho_{u^{\prime} u^{\prime}}$ and $\rho_{\theta^{\prime} \theta^{\prime}}$ are in the range of $800 \nu / u_{\tau}$. This is comparable to the generally accepted value $\left(1000 \nu / u_{\tau}\right)$ of the length of sublayer streaks Sreenivasan (1989). A careful observation of figures $7 \mathrm{a}$. $7 \mathrm{c}, 8 \mathrm{a}, 8 \mathrm{c}, 9 \mathrm{a}$, and $9 \mathrm{c}$ evokes that the structure which is responsible of moving 
streamwise kinetic energy in the buffer region can transport most of the scalar as well. If all the geometrical information such as angle of inclination, streamwise length, and spanwise width of $\rho_{u^{\prime} u^{\prime}}$ and $\rho_{\theta^{\prime} \theta^{\prime}}$ only in the buffer region are taken into consideration, it can be concluded that the structure that is responsible for transporting most of the streamwise kinetic energy as well as scalar fluctuations are streaks. However, this structural similarity is disturbed in the log layer and in the outer layer. This in turn suggests the distinct difference of the complete picture of the structure. Interestingly, no sign of similarity is detected between $\rho_{v^{\prime} v^{\prime}}$ and $\rho_{\theta^{\prime} \theta^{\prime}}$ at any of the levels of boundary layer. This implies that the complete structure which is carrying most of the kinetic energy is not exactly the same as the one that transport scalar. Despite the structure which is a reminiscent of a streak is largely responsible for carrying most of the streamwise turbulent kinetic energy and scalar fluctuations in the near-wall region, different forms of structures take the task over at the log layer and the outer layer. Hence, it is evident that LSM and very large scale motions (VLSM) which are responsible for transporting turbulent kinetic energy in the outer region are not exactly the same as that of carrying most of the scalar fluctuations.

(a)

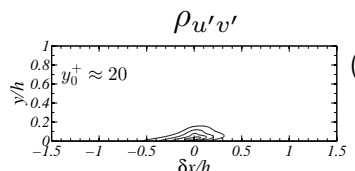

(d)

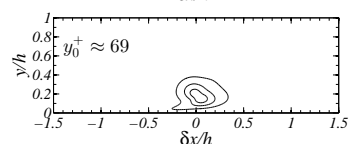

(g)

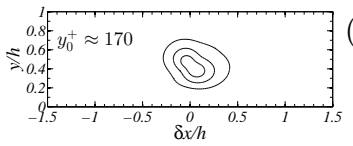

(b)
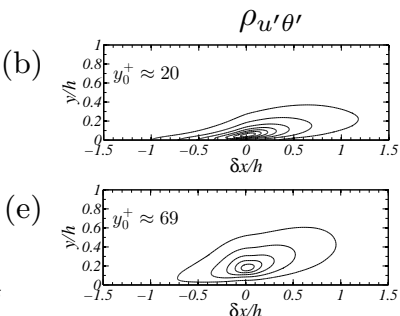

(h)

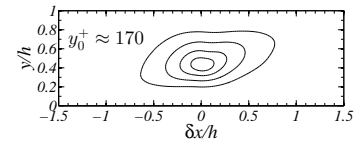

(c)

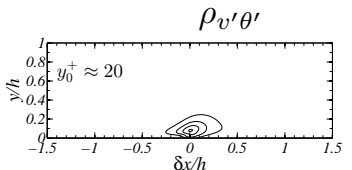

(f)

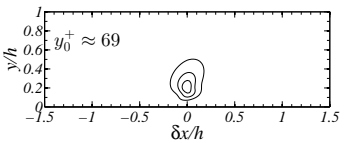

(i)

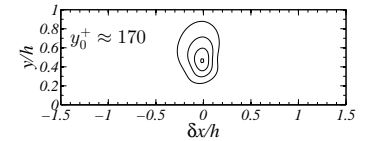

Figure 10: Two-dimensional sections of $\rho_{u^{\prime} v^{\prime}}$ in (a) (d) and (g) of $\rho_{u^{\prime} \theta^{\prime}}$ in (b) (e) and (h) of $\rho_{v^{\prime} \theta^{\prime}}$ in (c) (f) and (i) on streamwise wall-normal plane. Reference point is located at: $y_{0} / h \approx 0.054\left(y_{0}^{+} \approx 20\right)$ for $(\mathrm{a}) \cdot(\mathrm{c}) y_{0} / h \approx 0.18\left(y_{0}^{+} \approx 69\right)$ for $(\mathrm{d})(\mathrm{f})$ and $y_{0} / h \approx 0.45\left(y_{0}^{+} \approx 170\right)$ for (g) (i) Contour levels are from 0.2 with the increment of 0.1 . 
Figure 10 depicts the wall-normal streamwise view of $\rho_{u^{\prime} v^{\prime}}, \rho_{u^{\prime} \theta^{\prime}}$, and $\rho_{v^{\prime} \theta^{\prime}}$. Both $\rho_{u^{\prime} v^{\prime}}$ and $\rho_{u^{\prime} \theta^{\prime}}$ show negative values in the lower half of the channel. Positive values of $v^{\prime}$ are accompanied by negative values of $u^{\prime}$ and negatively valued $v^{\prime}$ s are associated with positively valued $u^{\prime}$ s owing to the near-wall turbulence phenomena. Hence, negative correlation between $v^{\prime}$ and $u^{\prime}$ is dominant. The hot lower wall boundary conditions impose negative correlation between $u^{\prime}$ and $\theta^{\prime}$. A positive correlation between the same variables was detected at the upper wall (not shown here). Structure of $\rho_{u^{\prime} \theta^{\prime}}$ significantly larger than that of both $\rho_{v^{\prime} \theta^{\prime}}$ and $\rho_{u^{\prime} v^{\prime}}$ as shown previously. It is interesting to note the close correspondence among $\rho_{u^{\prime} u^{\prime}}, \rho_{\theta^{\prime} \theta^{\prime}}$, and $\rho_{u^{\prime} \theta^{\prime}}$ (see $7 \mathrm{a}, 7 \mathrm{c}$, and $10 \mathrm{~b}$ respectively). Moreover, $u^{\prime}$ and $\theta^{\prime}$ are strongly correlated than any other cross-correlation in the buffer region because streamwise velocity field convects the thermal field downstream. The structure formed by $\rho_{u^{\prime} \theta^{\prime}}$ is apparently equivalent to the overlap region formed by $\rho_{u^{\prime} u^{\prime}}$ and $\rho_{\theta^{\prime} \theta^{\prime}}$. This implies that the structure that resembles a streak is also responsible for transporting streamwise heat flux in the viscous sublayer. The structure of $\rho_{u^{\prime} v^{\prime}}$ seems to be a scaled down version of $\rho_{u^{\prime} \theta^{\prime}}$ only in the viscous sublayer. This implies that the structure which is transporting Reynolds stresses are much smaller than the structure responsible of moving streamwise heat flux. It is interesting to note the close correspondence between the angle of inclination of $\rho_{u^{\prime} \theta^{\prime}}$ (see figures 10b, 10e, and 10h) and that of $\rho_{u^{\prime} u^{\prime}}$ 380 (see figures $7 \mathrm{a}, 7 \mathrm{~d}$, and $7 \mathrm{~g}$ ) at all the levels of the boundary layer. It can be seen that the structure of $\rho_{u^{\prime} \theta^{\prime}}$ is the dominant feature of transporting heat flux at all the levels of turbulent boundary layer. Much profound conclusion can be stated after observing all the directional aspects in the following figures. 
(a)
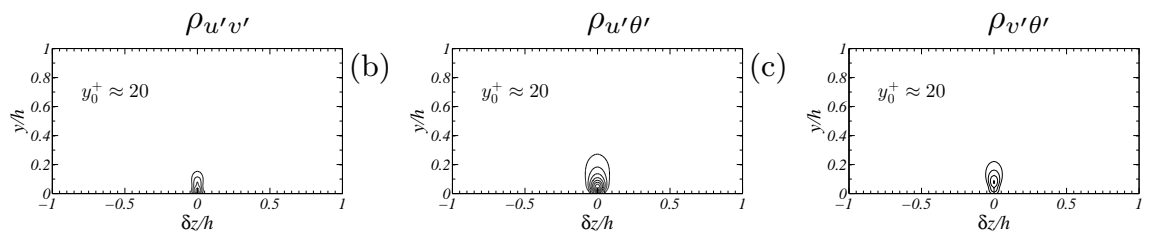

(d)
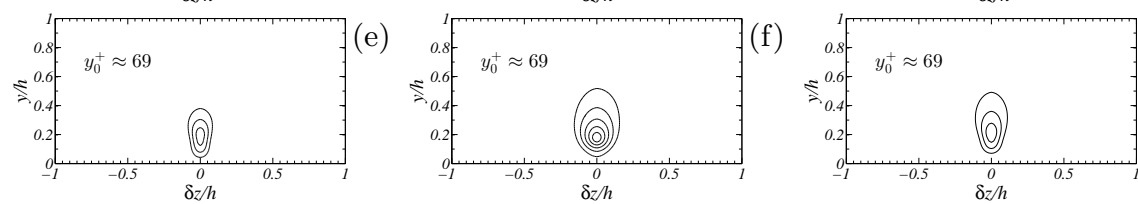

(g)
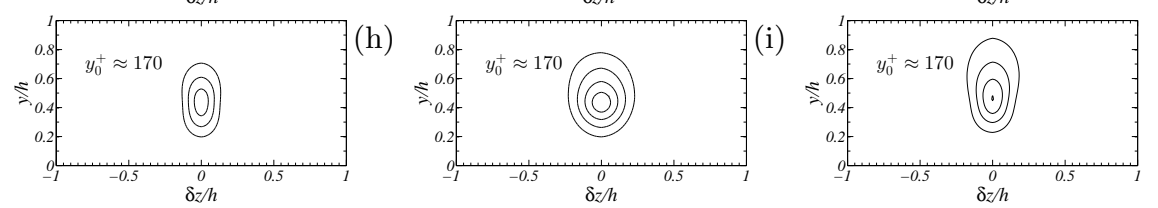

Figure 11: Two-dimensional sections of $\rho_{u^{\prime} v^{\prime}}$ in (a) (d) and (g) of $\rho_{u^{\prime} \theta^{\prime}}$ in (b) (e), and (h) of $\rho_{v^{\prime} \theta^{\prime}}$ in (c) (f) and (i) on spanwise wall-normal plane. Reference point is located at: $y_{0} / h \approx 0.054\left(y_{0}^{+} \approx 20\right)$ for (a) $[(\mathrm{c})] y_{0} / h \approx 0.18\left(y_{0}^{+} \approx 69\right)$ for $(\mathrm{d})\left[(\mathrm{f})\right.$ and $y_{0} / h \approx 0.45\left(y_{0}^{+} \approx 170\right)$ for (g) (i) Contour levels are from 0.2 with the increment of 0.1.

Figure 11 illustrates the spanwise wall-normal sectional view of $\rho_{u^{\prime} v^{\prime}}, \rho_{u^{\prime} \theta^{\prime}}$, and $\rho_{v^{\prime} \theta^{\prime}}$. It is interesting to note that spanwise sectional similarity of $\rho_{u^{\prime} v^{\prime}}$ and $\rho_{v^{\prime} \theta^{\prime}}$ besides the less correspondence between them denoted in the streamwise wall-normal cross sections. Lyons et al. (1991) concluded that the same eddies that control Reynolds stresses also govern turbulent heat transfer by superficially observing $\rho_{u^{\prime} v^{\prime}}$ and $\rho_{v^{\prime} \theta^{\prime}}$ only on spanwise wall-normal plane. However, close examination of all two dimensional planes in three mutually perpendicular directions projects a complete different picture. The structure of $\rho_{u^{\prime} \theta^{\prime}}$ is clearly the dominant feature on this plane as well. 
(a)

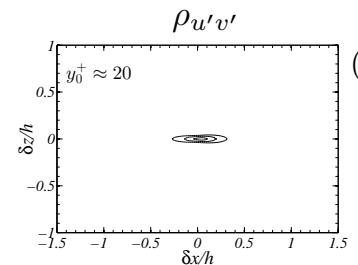

(d)

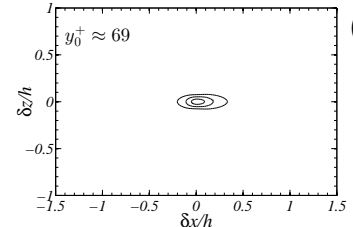

(g)

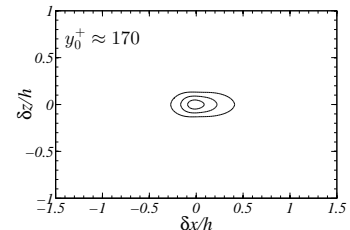

(b)

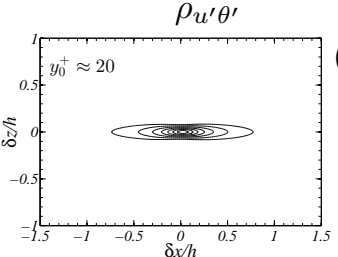

(e)

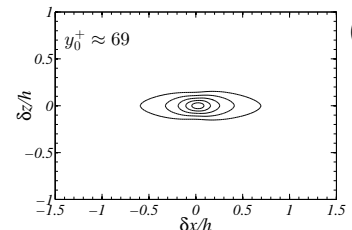

(h)

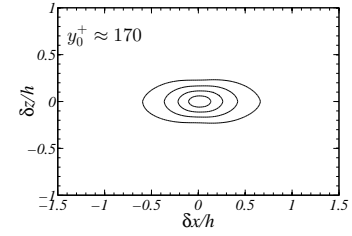

(c)

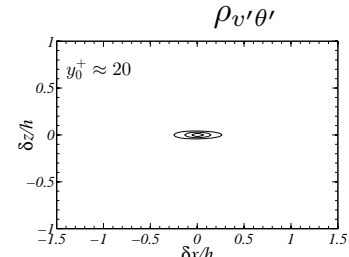

(f)

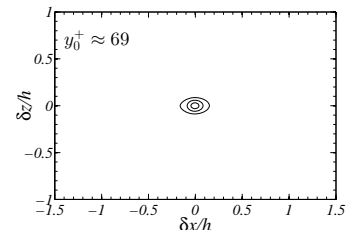

(i)

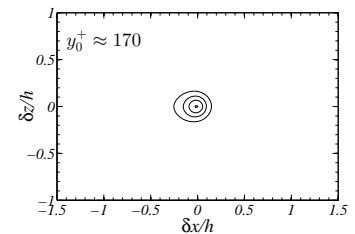

Figure 12: Two-dimensional sections of $\rho_{u^{\prime} v^{\prime}}$ in (a) (d) and (g) of $\rho_{u^{\prime} \theta^{\prime}}$ in (b) (e), and (h) of $\rho_{v^{\prime} \theta^{\prime}}$ in (c) (f), and (i) on spanwise streamwise plane. Reference point is located at: $y_{0} / h \approx 0.054\left(y_{0}^{+} \approx 20\right)$ for (a) $(\mathrm{c}) y_{0} / h \approx 0.18\left(y_{0}^{+} \approx 69\right)$ for $(\mathrm{d})\left[(\mathrm{f})\right.$ and $y_{0} / h \approx 0.45\left(y_{0}^{+} \approx 170\right)$ for (g) (i) Contour levels are from 0.2 with the increment of 0.1

Figure 12 presents the sectional views of $\rho_{u^{\prime} v^{\prime}}, \rho_{u^{\prime} \theta^{\prime}}$, and $\rho_{v^{\prime} \theta^{\prime}}$ on streamwise spanwise plane. Streamwise length scales of $\rho_{u^{\prime} v^{\prime}}$ and $\rho_{v^{\prime} \theta^{\prime}}$ show that structure that contains most of the Reynolds stress is significantly different than the structure that contribute most of the wall normal heat flux. Previous observations contrasts the conclusion of Lyons et al. (1991) which was only based on spanwise wall-normal views of $\rho_{u^{\prime} v^{\prime}}$ and $\rho_{u^{\prime} v^{\prime}}$. Additionally, if figures $12 \mathrm{~b}, 12 \mathrm{e}$ and $12 \mathrm{~h}$ are observed, it is readily seen the dominance of $\rho_{u^{\prime} \theta^{\prime}}$ structure in all the regions of boundary layer. Therefore, it can be concluded that the structure of $\rho_{u^{\prime} \theta^{\prime}}$ is the dominant large scale feature of heat transport in wall bounded turbulence. And the structure of $\rho_{u^{\prime} v^{\prime}}$ has little correspondence with both $\rho_{u^{\prime} \theta^{\prime}}$ structure and $\rho_{v^{\prime} \theta^{\prime}}$ structure. 


\subsection{Length scales of structures}

\begin{tabular}{llllll}
\hline \hline$\rho_{\alpha^{\prime} \beta^{\prime}}$ & $y_{0}^{+}$ & $L_{x} / h$ & $L_{y} / h$ & $L_{z} / h$ & $V_{\text {box }} / h^{3}$ \\
\hline$\rho_{u^{\prime} u^{\prime}}$ & 20 & 2.20 & 0.250 & 0.20 & 0.110 \\
& 69 & 2.30 & 0.475 & 0.30 & 0.328 \\
\hline & 170 & 2.30 & 0.700 & 0.50 & 0.805 \\
$\rho_{v^{\prime} v^{\prime}}$ & 20 & 0.55 & 0.200 & 0.10 & 0.011 \\
& 69 & 0.40 & 0.350 & 0.20 & 0.028 \\
\hline & 170 & 0.45 & 0.600 & 0.30 & 0.081 \\
$\rho_{\theta^{\prime} \theta^{\prime}}$ & 20 & 1.80 & 0.225 & 0.20 & 0.081 \\
& 69 & 1.50 & 0.425 & 0.40 & 0.255 \\
\hline & 170 & 1.20 & 0.775 & 0.60 & 0.558 \\
$\rho_{u^{\prime} v^{\prime}}$ & 20 & 0.90 & 0.150 & 0.10 & 0.014 \\
& 69 & 0.55 & 0.325 & 0.20 & 0.036 \\
\hline & 170 & 0.70 & 0.500 & 0.30 & 0.105 \\
\hline$\rho_{u^{\prime} \theta^{\prime}}$ & 20 & 2.20 & 0.275 & 0.20 & 0.121 \\
& 69 & 1.70 & 0.475 & 0.30 & 0.242 \\
\hline & 170 & 1.45 & 0.600 & 0.45 & 0.391 \\
\hline \hline$v_{v^{\prime} \theta^{\prime}}$ & 69 & 0.40 & 0.425 & 0.20 & 0.017 \\
& 170 & 0.45 & 0.650 & 0.35 & 0.102 \\
\hline \hline
\end{tabular}

Table 2: Streamwise length $\left(L_{x}\right)$, wall-normal length $\left(L_{y}\right)$, spanwise length $\left(L_{z}\right)$, and volume of the circumscribed box $\left(L_{x} . L_{y} . L_{z}=V_{b o x}\right)$ around the object of $\rho_{u^{\prime} u^{\prime}}, \rho_{v^{\prime} v^{\prime}}, \rho_{\theta^{\prime} \theta^{\prime}} \rho_{u^{\prime} v^{\prime}}, \rho_{u^{\prime} \theta^{\prime}}$, and $\rho_{v^{\prime} \theta^{\prime}}$ structures. All dimensions are normalized in outer coordinates.

A concise summary of streamwise, wall-normal, and spanwise lengths of $\rho_{u^{\prime} u^{\prime}}, \rho_{v^{\prime} v^{\prime}}, \rho_{\theta^{\prime} \theta^{\prime}}, \rho_{u^{\prime} v^{\prime}}, \rho_{u^{\prime} \theta^{\prime}}$, and $\rho_{v^{\prime} \theta^{\prime}}$ is given in table 2. Each length scale $L_{x}, L_{y}, L_{z}$ is the longest spatial extent of structure measured by using the relevant two dimensional views of correlations maps. Since it can be heuristically 
assumed that the relative content of energy or amount of flux is proportional to the volume of the structure, $V_{b o x}$ is computed by evaluating the volume of the circumscribed box (i.e, $L_{x} \cdot L_{y} \cdot L_{z}$ ) around the iso-surface of the correlation map. The quantity $V_{\text {box }}$ provides an overall sense of the size of the structure as well. Figures 13a, 13b, 13c, 13d shows the growth of each structure in terms of their streamwise length $\left(L_{x}\right)$, wall-normal length $\left(L_{y}\right)$, spanwise length $\left(L_{z}\right)$, and the volume $V_{\text {box }}$ respectively. These figures illustrate the growth of the structures with respect to the location at which the computation of the correlation based on and provide a quick assessment of data in table 2.

(a)

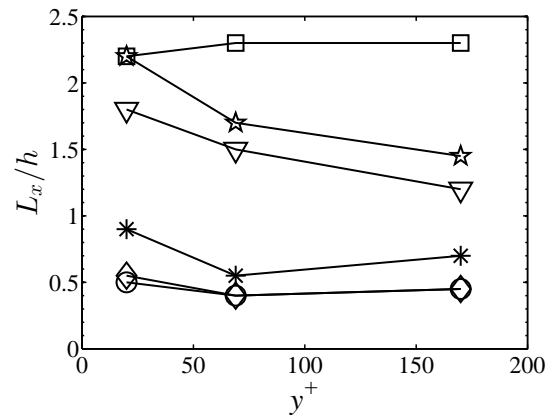

(c)

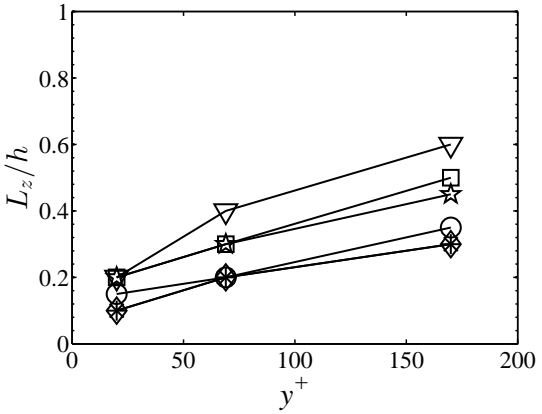

(b)

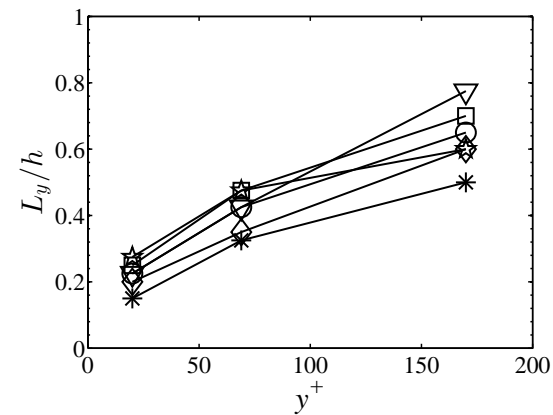

(d)

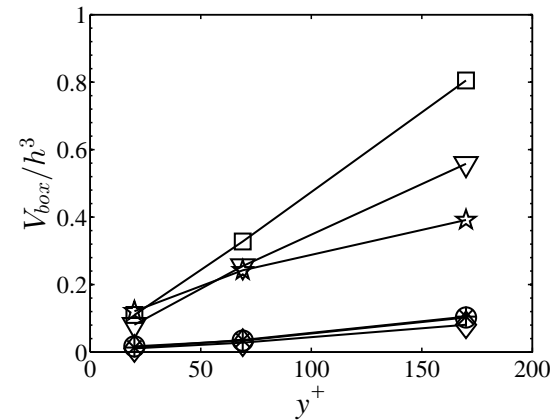

Figure 13: (a) Streamwise length $\left(L_{x}\right)$, (b) wall-normal length $\left(L_{y}\right)$, (c) spanwise length $\left(L_{z}\right)$, and (d) volume of the circumscribed box around the object of $\rho_{u^{\prime} u^{\prime}}(\square), \rho_{v^{\prime} v^{\prime}}(\diamond), \rho_{\theta^{\prime} \theta^{\prime}}(\nabla)$, $\rho_{u^{\prime} v^{\prime}}(*), \rho_{u^{\prime} \theta^{\prime}}\left(\right.$ گ) , and $\rho_{v^{\prime} \theta^{\prime}}(\mathrm{O})$.

The largest structure in the streamwise direction is $\rho_{u^{\prime} u^{\prime}}$. According to the figure $13 \mathrm{c}$ the spanwise extent of $\rho_{\theta^{\prime} \theta^{\prime}}$ takes over that of $\rho_{u^{\prime} u^{\prime}}$ specifically beyond the buffer region. However, the overall size of $\rho_{\theta^{\prime} \theta^{\prime}}$ is smaller than that of $\rho_{u^{\prime} u^{\prime}}$ as seen in figure $13 \mathrm{~d}$. Interestingly, structures of $\rho_{u^{\prime} u^{\prime}}$ and $\rho_{\theta^{\prime} \theta^{\prime}}$ show a linear growth 
as per figure $13 \mathrm{~d}$. This reassures that scalar fluctuations are advected by the streamwise velocity fluctuations confirming the previous observation based on three and two dimensional correlation maps. It can be seen that the streamwise lengths of both $\rho_{\theta^{\prime} \theta^{\prime}}$ and $\rho_{u^{\prime} \theta^{\prime}}$ reduce as they move away from the wall (see figure 13a Coincidentally, according to the figure $3 \mathrm{a}$ a local minimum of $\theta_{r m s}^{+}$ is observed at the vicinity of $y^{+}=70$. This might cause streamwise length of $\rho_{\theta^{\prime} \theta^{\prime}}$ and $\rho_{u^{\prime} \theta^{\prime}}$ to become shorter in the buffer region. However, their spanwise lengths, wall-normal lengths, and the volume of the boxes increase away from the wall. This suggests that the influence of streamwise velocity fluctuations on scalar transport diminishes beyond the buffer region. Streamwise length scales of $\rho_{u^{\prime} v^{\prime}}, \rho_{v^{\prime} v^{\prime}}$, and $\rho_{v^{\prime} \theta^{\prime}}$ are order of magnitude lower than that of other correlations shown in figure 13a. Relatively weak wall-normal velocity fluctuations cause correlations to die out faster resulting shorter length scales. However, $\rho_{u^{\prime} v^{\prime}}, \rho_{v^{\prime} v^{\prime}}$, and $\rho_{v^{\prime} \theta^{\prime}}$ show linear volumetric growth as shown in figure 13d. It is interesting to notice that the size of all the correlations but $\rho_{u^{\prime} \theta^{\prime}}$ demonstrate a linear growth across the boundary layer. The growth rates of structures are distinct to each other except for the structures that are responsible of Reynolds stresses and wall-normal heat fluxes. This propose that not only the spatial extent of the structures which are responsible for momentum and scalar transport but also their growth rates are distinct to each other.

\subsection{Reynolds analogy}

The similarity of the length in all three mutually perpendicular directions of strucutures of $\rho_{v^{\prime} v^{\prime}}$ and $\rho_{v^{\prime} \theta^{\prime}}$ is very strong throughout the boundary layer.

445 Both streamwise and wall normal lengths of the LSM of Reynolds stress are not exactly the same as those of the LSM of wall-normal heat flux. However, their spanwise lengths show a close correspondence especially in the log region. Moreover, the similarity of circumscribed boxes of $\rho_{v^{\prime} v^{\prime}}$ and $\rho_{v^{\prime} \theta^{\prime}}$ is remarkable (see 13d). This behavior of LSM can be used to elicit the validity of Reynolds' analogy in LSM point of view. According to Tennekes \& Lumley (1972), in a pure shear flow in which only $y$ is the direction of non-homogeneity, eddy 
viscosity $\left(\nu_{t}\right)$ and eddy diffusivity $\left(\alpha_{t}\right)$ can be defined as,

$$
-\overline{u^{\prime} v^{\prime}}=\nu_{t} \frac{\partial U}{\partial y}
$$

and

$$
\overline{v^{\prime} \theta^{\prime}}=-\alpha_{t} \frac{\partial \Theta}{\partial y}
$$

respectively. This follows the definition of $P r_{t}$ as

$$
\operatorname{Pr}_{t}=\frac{\nu_{t}}{\alpha_{t}}=\frac{-\overline{u^{\prime} v^{\prime}}(\partial \Theta / \partial y)}{\overline{v^{\prime} \theta^{\prime}}(\partial U / \partial y)}
$$

Figure 14 shows the variation of $P r_{t}$ across the boundary layer. Two plateaus, one in the inner region and the other in the outer region of the boundary layer, are detected in the variation of the $P r_{t}$ across the boundary layer. Note that $\mathrm{Pr}_{t}$ is slightly higher than one in the inner region while it is slightly lower than one in the outer region. It is the value of $P r_{t}$ which is most influential quantity in determining the relative importance of Reynolds stresses and wall-normal heat fluxes in wall-bounded turbulence.

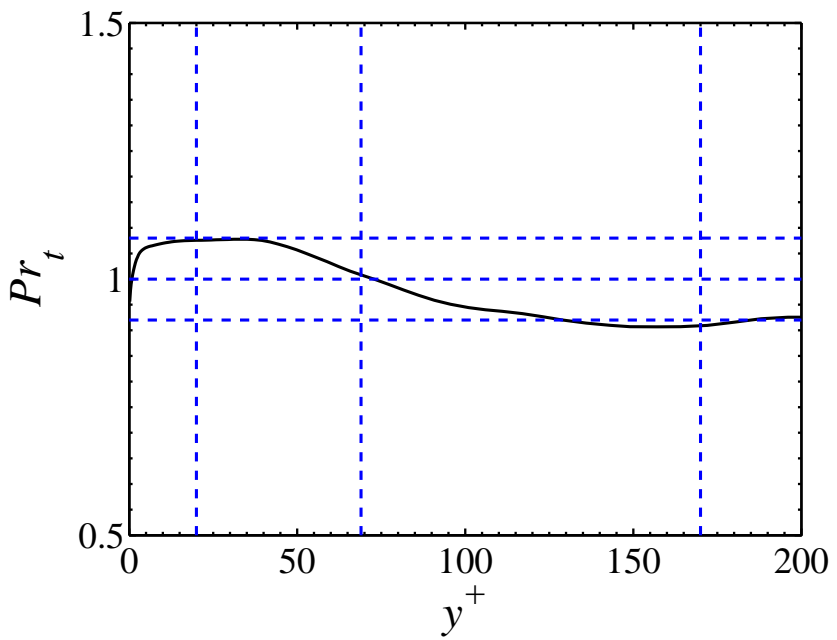

Figure 14: Profile of turbulent Prandtl number $\left(P r_{t}\right)$. Vertical blue dashed-lines are drawn at locations where $y^{+}=20, y^{+}=69$, and $y^{+}=170$ from left to right respectively. Horizontal dashed-lines represents $\operatorname{Pr}_{t}=0$ and \pm 1.08 from top to bottom respectively.

It can be readily notice from figure 13 a that streamwise lengths of LSM of Reynolds stress are higher than that of wall-normal heat fluxes following the 
trend of $\mathrm{Pr}_{t}$ in the near wall region. Streamwise lengths of LSM of the same quantities are approximately the same in the log regions. This is consistent with the value of $\operatorname{Pr}_{t}$ detected in the log region according to figure 14 . However, streamwise lengths do not quiet follow the trend of $\operatorname{Pr}_{t}$ at the outer region. Wall-normal and spanwise lengths of LSM of both Reynolds stress and wallnormal heat flux more or less follow the trend of $P r_{t}$ in both inner and outer regions of the boundary layer. This suggests that Reynolds analogy holds for the scalar transport by LSM in near-wall turbulence.

\section{Conclusions}

In the present study, DNS database of a thermal turbulent channel flow at $R e_{\tau}=395$ is used to investigate the influence of large scale motions on the scalar transport and to identify whether the structures that are formed by scalar fluctuations are similar to the structures that are created by velocity fluctuations.

Striking resemblance between $\rho_{u^{\prime} u^{\prime}}$ and $\rho_{\theta^{\prime} \theta^{\prime}}$ was detected only in the viscous sublayer. The structural similarity is so strong that it is readily seen in all three mutually perpendicular directions. These near-wall structures of both $\rho_{u^{\prime} u^{\prime}}$ and $\rho_{\theta^{\prime} \theta^{\prime}}$ seems to be reminiscent of the long thin streamwise streaks observed in the viscous sublayer. In fact, it was shown that length scales of $\rho_{u^{\prime} u^{\prime}}$ and $\rho_{\theta^{\prime} \theta^{\prime}}$ structures have the same orders of magnitudes of the streamwise streaks. Moreover, $\rho_{u^{\prime} \theta^{\prime}}$ structure is much akin to structure of both $\rho_{u^{\prime} u^{\prime}}$ and $\rho_{\theta^{\prime} \theta^{\prime}}$ in the same region of the boundary layer. Thus, it can be inferred that organized motions which are responsible of transporting streamwise component of the turbulent kinetic energy also carry most of the scalar fluctuation as well as streamwise heat flux only in the viscous sublayer. This is consistent with the high correlation observed between streamwise velocity fluctuations and scalar fluctuations by Kim \& Moin (1987) and remarkable resemblance between high and low speed streaks and low temperature had high temperature thermal streaks detected by Iritani et al. (1985), Kim \& Moin (1987), and Kong et al. (2000). 
It is seen that the structure of LSM of $\rho_{u^{\prime} u^{\prime}}$ is completely different from that of $\rho_{\theta^{\prime} \theta^{\prime}}$ in the log layer and in the outer layer. This suggests that LSM that carry most of the turbulent kinetic energy is completely distinct from LSM which are responsible of transporting scalar in the log layer and in the outer layer. However, it is worthwhile to note the influence of imposed scalar boundary conditions. Notice the close similarity between the profile of $\overline{u^{\prime 2}}$ and that of $\theta_{r m s}^{+}$in the near wall region and the dissimilarity of them beyond $y^{+}=50$. This is merely a consequence of the iso-thermal boundary condition in the channel flow. The outer peak observed in the profile of $\theta_{r m s}^{+}$does not occur when iso-flux boundary condition is imposed. Therefore it is important to bare in mind that this is a limitation of the present study and its conclusions. Interestingly, the slight similarity detected between the structure of $\rho_{u^{\prime} u^{\prime}}$ and that of $\rho_{u^{\prime} \theta^{\prime}}$ indicates that LSM which transport streamwise component of the turbulent kinetic energy is partially analogous LSM which move streamwise heat flux. Moreover, the structure of $\rho_{u^{\prime} \theta^{\prime}}$ is the dominant large scale structure that transport streamwise heat flux across the boundary layer.

However, there is no correspondence between the organized motions of $\rho_{u^{\prime} v^{\prime}}$ and $\rho_{u^{\prime} \theta^{\prime}}$ either within or beyond the viscous sublayer. Therefore, it is deduced that the structure of LSM which promote Reynolds stress transport is completely different from that of the LSM take part in carrying scalar fluxes. An extensive analysis of length scales of structures in three mutually perpendicular directions revealed that the growth of LSM which are reponsible of both momentum and scalar transport is linear. However, they demonstrate different rates of growth with the highest rate is observed in LSM that carry most of the streamwise turbulent kinetic energy. Finally but most importantly, the comparison of physical extents of LSM which are responsible for transporting Reynolds stresses and wall-normal heat fluxes reveals that Reynolds analogy 


\section{References}

Abe, H., Antonia, R. A., \& Kawamura, H. (2009). Correlation between smallscale velocity and scalar fluctuations in a turbulent channel flow. Journal of Fluid Mechanics, 627, 1-32.

Abe, H., Kawamura, H., \& Matsuo, Y. (2001). Direct numerical simulation of a fully developed turbulent channel flow with respect to the reynolds number dependence. Journal of fluids Engineering, 123, 382-393.

Adrian, R. (2007). Hairpin vortex organization in wall turbulencea). Physics of Fluids, 19, 041301.

530 del Álamo, J. C., \& Jiménez, J. (2003). Spectra of the very large anisotropic scales in turbulent channels. Physics of Fluids, 15, L41-L44.

del Álamo, J. C., \& Jiménez, J. (2006). Linear energy amplification in turbulent channels. Journal of Fluid Mechanics, 559, 205-213.

Antonia, R., Abe, H., \& Kawamura, H. (2009). Analogy between velocity and scalar fields in a turbulent channel flow. Journal of Fluid Mechanics, 628, $241-268$.

Araya, G., Leonardi, S., \& Castillo, L. (2008). Numerical assessment of local forcing on the heat transfer in a turbulent channel flow. Physics of Fluids, 20.

Bakewell, H. P., \& Lumley, J. L. (1967). Viscous sublayer and adjacent wall region in turbulent pipe flow. Physics of Fluids (1958-1988), 10, 1880-1889.

Balakumar, B., \& Adrian, R. (2007). Large- and very-large-scale motions in channel and boundary-layer flows. Philosophical Transactions of the Royal Society of London A: Mathematical, Physical and Engineering Sciences, 365, 665-681.

Blackwelder, R. F., \& Eckelmann, H. (1979). Streamwise vortices associated with the bursting phenomenon. Journal of Fluid Mechanics, 94, 577-594. 
Corrsin, S. (1956). Some corrent problems in turbulent shear flows. In F. S. Sherman (Ed.), Proceedings of First Naval Hydrodynamic symposium (pp. $373-400)$.

Guala, M., Hommema, S. E., \& Adrian, R. J. (2006). Large-scale and verylarge-scale motions in turbulent pipe flow. Journal of Fluid Mechanics, 554, $521-542$.

Head, M. R., \& Bandyopadhyay, P. (1981). New aspects of turbulent boundarylayer structure. Journal of Fluid Mechanics, 107, 297-338.

Hoyas, S., \& Jimnez, J. (2006). Scaling of the velocity fluctuations in turbulent channels up to re=2003. Physics of Fluids, 18, 011702.

Hussain, A. K. M. F. (1986). Coherent structures and turbulence. Journal of Fluid Mechanics, 173, 303-356.

Hutchins, N., \& Marusic, I. (2007a). Large scale influences in near-wall turbulence. Phil. Trans. R. Soc. A, 365, 647-664.

Hutchins, N., \& Marusic, I. (2007b). Evidence of very long meandering features in the logarithmic region of turbulent boundary layers. Journal of Fluid Mechanics, 579, 1-28.

Iritani, Y., Kasagi, N., \& Hirata, M. (1985). Heat transfer mechanism and associated turbulence structure in the near-wall region of a turbulent boundary layer. In L. Bradbury, F. Durst, B. E. Launder, F. W. Schmidt, \& J. H. Whitelaw (Eds.), Turbulent Shear Flows 4 (pp. 223-234). Springer Berlin Heidelberg. Research Briefs, 137, 54.

Johansson, A., \& Wikström, P. (1999). Dns and modelling of passive scalar transport in turbulent channel flow with a focus on scalar dissipation rate modelling. Flow, Turbulence and Combustion, 63, 223-245. 
575 Kasagi, N., \& Iida, O. (1999). Progress in direct numerical simulation of turbulent heat transfer. In Proceedings of the 5th ASME/JSME Joint Thermal Engineering Conference.

Kim, J., \& Moin, P. (1985). Application of a fractional-step method to incompressible navier-stokes equations. Journal of Computational Physics, 59, 308 $-323$.

Kim, J., \& Moin, P. (1987). Transport of passive scalars in a turbulent channel flow. Tecnical Memorandum NASA TM-89463 NASA Ames Research Center Moffett Field, California 94035.

Kim, K. C., \& Adrian, R. J. (1999). Very large-scale motion in the outer layer. Physics of Fluids, 11, 417-422.

Kline, S. J., Reynolds, W. C., Schraub, F. A., \& Runstadler, P. W. (1967). The structure of turbulent boundary layers. Journal of Fluid Mechanics, 30, $741-773$.

Kong, H., Choi, H., \& Lee, J. S. (2000). Direct numerical simulation of turbulent thermal boundary layers. Physics of Fluids (1994-present), 12, 2555-2568.

Kong, H., Choi, H., \& Lee, J. S. (2001). Dissimilarity between the velocity and temperature fields in a perturbed turbulent thermal boundary layer. Physics of Fluids (1994-present), 13, 1466-1479.

Kovasznay, L. S. G., Kibens, V., \& Blackwelder, R. F. (1970). Large-scale motion in the intermittent region of a turbulent boundary layer. Journal of Fluid Mechanics, 41, 283-325.

Lee, J. H., \& Sung, H. J. (2011). Very-large-scale motions in a turbulent boundary layer. Journal of Fluid Mechanics, 673, 80-120.

Liu, Z., Adrian, R. J., \& Hanratty, T. J. (2001). Large-scale modes of turbulent 600 channel flow: transport and structure. Journal of Fluid Mechanics, 448, 53-80. 
Lyons, S., Hanratty, T., \& McLaughlin, J. (1991). Direct numerical simulation of passive heat transfer in a turbulent channel flow. International Journal of Heat and Mass Transfer, 34, 1149 - 1161.

Mathis, R., Hutchins, N., \& Marusic, I. (2009). Large-scale amplitude modulation of the small-scale structures in turbulent boundary layers. Journal of Fluid Mechanics, 628, 311-337.

Moin, P., \& Mahesh, K. (1998). Direct numerical simulation: A tool in turbulence research. Annual Review of Fluid Mechanics, 30, 539-578.

Moser, R. D., Kim, J., \& Mansour, N. N. (1999). Direct numerical simulation of turbulent channel flow up to re=590. Phys. Fluids, 11, 943-945.

Orlandi, P. (2000). Fluid Flow Phenomena: A Numerical Toolkit volume 55 of Fluid Mechanics and its Applications. Kluwer Academic Publishers.

Orlandi, P., \& Jiménez, J. (1994). On the generation of turbulent wall friction. Physics of Fluids, 6, 634-641.

Panton, R. L. (2001). Overview of the self-sustaining mechanisms of wall turbulence. Progress in Aerospace Sciences, 37, 341 - 383.

Perry, A. E., \& Hoffmann, P. H. (1976). An experimental study of turbulent convective heat transfer from a flat plate. Journal of Fluid Mechanics, 77, $355-368$.

Robinson, S. K. (1991). Coherent motions in the turbulent boundary layer. Annual Review of Fluid Mechanics, 23, 601-639.

Shraiman, B. I., \& Sigga, E. D. (2000). Scalar turbulence. Nature, 405, 639-646.

Sillero, J. A., Jimnez, J., \& Moser, R. D. (2014). Two-point statistics for turbulent boundary layers and channels at reynolds numbers up to +2000 . Physics of Fluids (1994-present), 26, 105109. 
Smith, C. R., Walker, J. D. A., Haidari, A. H., \& Sobrun, U. (1991). On the dynamics of near-wall turbulence. Philosophical Transactions: Physical Sciences and Engineering, 336, pp. 131-175.

Smits, A. J., McKeon, B. J., \& Marusic, I. (2011). Highreynolds number wall turbulence. Annual Review of Fluid Mechanics, 43, 353-375.

Sreenivasan, K. (1989). The turbulent boundary layer. In M. Gad-el Hak (Ed.), Frontiers in Experimental Fluid Mechanics (pp. 159-209). Springer Berlin Heidelberg volume 46 of Lecture Notes in Engineering.

Sreenivasan, K. R. (1991). On local isotropy of passive scalars in turbulent shear flows. Proceedings of the Royal Society of London. Series A: Mathematical and Physical Sciences, 434, 165-182.

Teitel, M., \& Antonia, R. (1993). Heat transfer in fully developed turbulent channel flow: comparison between experiment and direct numerical simulations. International Journal of Heat and Mass Transfer, 36, 1701 - 1706.

Tennekes, H., \& Lumley, J. L. (1972). A First Course in Turbulence. The MIT Press.

Townsend, A. A. (1956). The Structure of Turbulent Shear Flow. (1st ed.). Cambridge University Press.

${ }_{645}$ Warhaft, Z. (2000). Passive scalars in turbulent flows. Annual Review of Fluid Mechanics, 32, 203-240. 